\title{
Body Mass Index and Depressive Symptoms: Testing for Adverse and Protective Associations in Two Twin Cohort Studies
}

\author{
Markus Jokela, ${ }^{1}$ Venla Berg, ${ }^{1}$ Karri Silventoinen, ${ }^{2}$ G. David Batty, ${ }^{3,4,5}$ Archana Singh-Manoux, ${ }^{3,6}$ \\ Jaakko Kaprio, ${ }^{7,8,9}, \uparrow$ George Davey Smith, ${ }^{10,11}, \uparrow$ and Mika Kivimäki ${ }^{3} \uparrow$ \\ ${ }^{1}$ Institute of Behavioral Sciences, University of Helsinki, Helsinki, Finland \\ ${ }^{2}$ Department of Social Research, University of Helsinki, Helsinki, Finland \\ ${ }^{3}$ Research Department of Epidemiology and Public Health, University College London, London, UK \\ ${ }^{4}$ Centre for Cognitive Ageing and Cognitive Epidemiology, Department of Psychology, University of Edinburgh, Scotland, \\ UK \\ ${ }^{5}$ Alzheimer Scotland Dementia Research Centre, Department of Psychology, University of Edinburgh, Scotland, UK \\ 'INSERM U1018, Centre for Research in Epidemiology \& Population Health, AP-HP, Paris, France \\ ${ }^{7}$ Department of Public Health, University of Helsinki, Helsinki, Finland \\ ${ }^{8}$ Department of Mental Health and Substance Abuse Services, National Institute for Health and Welfare, Helsinki, Finland \\ ${ }^{9}$ Institute for Molecular Medicine (FIMM), University of Helsinki, Helsinki, Finland \\ ${ }^{10}$ School of Social and Community Medicine, University of Bristol, Bristol, UK \\ ${ }^{11}$ MRC Integrative Epidemiology Unit, University of Bristol, Bristol, UK
}

Studies have suggested both adverse and protective associations of obesity with depressive symptoms. We examined the contribution of environmental and heritable factors in this association. Participants were same-sex twin pairs from two population-based twin cohort studies, the Older Finnish Twin Cohort ( $n=8,215$; mean age $=44.1$ ) and the US Midlife Development in the United States (MIDUS; $n=1,105$; mean age $=45.1$ ). Body mass index (BMI) was calculated from self-reported height and weight. Depressive symptoms were assessed using Beck's Depression Inventory (BDI; Finnish Twin Cohort), and by negative and positive affect scales (MIDUS). In the Finnish Twin Cohort, higher BMI was associated with higher depressive symptoms in monozygotic $(\mathrm{MZ})$ twins $(B=2.01,95 \% \mathrm{Cl}=1.0,3.0)$ and dizygotic $(\mathrm{DZ})$ twins $(B$ $=1.17,0.5,1.9)$ with $\mathrm{BMI}>22$. This association was observed in within-pair analysis in $\mathrm{DZ}$ twins $(B=1.47$, $\mathrm{Cl}=0.4,2.6)$ but not in within-pair analysis of $\mathrm{MZ}$ twins $(\mathrm{B}=0.03, \mathrm{Cl}=-1.9,2.0)$. Consistent with the latter result, a bivariate genetic model indicated that the association between higher $\mathrm{BMI}$ and higher depressive symptoms was largely mediated by genetic factors. The results of twin-pair analysis and bivariate genetic model were replicated in the MIDUS sample. These findings suggest an association between obesity and higher depressive symptoms, which is largely explained by shared heritable biological mechanisms.

Keywords: obesity, depression, twin study, epidemiology, mental health

Obese individuals have a higher risk of depression and anxiety (Blaine, 2008; Gariepy et al., 2010; Kivimäki et al., 2009; Luppino et al., 2010; Markowitz et al., 2008; Wardle et al., 2011). However, some studies have suggested that higher BMI might be associated with a lower rather than higher risk of depression (Crisp \& McGuiness, 1976; Gariepy et al., 2010; Lawlor et al., 2007; Palinkas et al., 1996). These conflicting findings may be due to unmeasured confounding factors.

In an attempt to obtain less confounded estimates, recent studies have used instrumental variables regression with genetic variants of body weight (Jokela et al., 2012; Kivimäki et al., 2011; Lawlor et al., 2011; Samaan et al.,
2013). The results from these studies have also been mixed, with both adverse (Jokela et al., 2012; Kivimäki et al., 2011) and protective (Lawlor et al., 2011) associations between BMI and depressive symptoms. Furthermore, allelic variation in the FTO gene - which is associated with higher

RECEIVED 22 February 2016; ACCEPTED 23 February 2016. First published online 8 April 2016.

ADDRESS FOR CORRESPONDENCE: Markus Jokela, Institute of Behavioural Sciences, Siltavuorenpenger 1A, PO Box 9, 00014 University of Helsinki, Finland. E-mail: markus.jokela@helsinki.fi

${ }^{\dagger}$ These authors contributed equally. 
obesity risk — has been associated with lower risk of depression (Samaan et al., 2013). Based on these genetic findings, it has been argued that the genetic and non-genetic pathways linking obesity and depression may be different (Lawlor et al., 2011).

In the present study, we applied twin-pair analysis and quantitative genetic modeling to test whether higher BMI is a risk or protective factor for depressive symptoms mediated differently via environmental and genetic pathways. Based on earlier studies, we hypothesized that there is an environmentally mediated risk but genetically mediated protective association between higher BMI and depressive symptoms.

\section{Method}

\section{The Older Finnish Twin Cohort Study}

The Older Finnish Twin Cohort study consists of all Finnish twin pairs of the same sex born before 1958 with both cotwins alive in 1975 (Kaprio \& Koskenvuo, 2002). Zygosity was assessed at baseline with questions about the similarity of appearance of a twin pair at an early school age, which has been shown to have high validity against genetic markers in this study (Kaprio \& Koskenvuo, 2002). With a response rate of $89 \%$, the cohort comprised of 13,888 twin pairs of known zygosity. The study was approved by the ethics committee of the Helsinki University hospital, and participants provided informed consent.

In a resurvey in 1990 of twins born 1930-1957, the participants were administered the self-reported BDI, which consists of 21 items each rated with a 4 -point scale $(0=$ no symptom, 3 = severe symptom; Beck \& Steer, 1987). To avoid conceptual overlap between BMI and depressive symptoms, two items referring to change in weight and appetite were excluded in the calculation of the total score in the present study (the inclusion or exclusion of these items did not have substantial influence on the results). The original scale of the 21-item inventory (ranging between 0 and 63) was retained by calculating the mean of the 19 items and multiplying this mean by 21 , excluding individuals with more than two missing items of the 19-item scale. Height and weight were self-reported in 1990, and BMI calculated as weight in kilograms/height in meters ${ }^{2}$. Covariates included were sex, age, education (reported on an 8 -point scale with a range $1=$ mandatory schooling to $8=$ university degree), and smoking (never-smoker, ex-smoker, current smoker).

\section{The Midlife Development in the United States (MIDUS) Twin Sample}

The MacArthur Foundation Survey of Midlife Development in the United States (MIDUS) is based on a nationally representative random-digit-dial sample of non-institutionalized, English-speaking adults aged 25 to 74 years in 1995-1996 (Brim et al., 2007). The estimated overall response rate was $60.8 \%$. The original sample of
7,108 participants includes a twin subsample $(n=1,908)$ of $715 \mathrm{MZ}$ twins, 671 same-sex DZ twins, 497 different-sex DZ twins, and 25 twins with unknown zygosity. Zygosity was determined using a self-reported questionnaire on the twin pair's similarity of eye and hair color and the similarity of appearance during childhood. Only same-sex DZ twin pairs were included in the present study. The survey complied with institutional review board standards of the University of Wisconsin and of the Harvard Medical School, and the study protocol was approved by the human study committees of both schools. All participants provided informed consent for participation.

In MIDUS, negative mood was assessed using a selfreported scale consisting of six items assessing positive affect (e.g., cheerful, full of life) and six items assessing negative affect (e.g., hopeless, so sad nothing could cheer you up) experienced during the last month rated on a 5-point scale $(1=$ none of the time, $5=$ all of the time $)$. These items were derived for the MIDUS questionnaire from six existing standardized scales of depressive symptoms and mental wellbeing (Mroczek \& Kolarz, 1998). The 12 items were summed together so that higher scores indicated negative mood (correlation between positive and negative affect scales $r=-0.63$ ). Self-reported height and weight were used to calculate BMI. Covariates included sex, age, education (reported on a 12-point scale ranging from $1=$ no schooling, 12 = higher education), and smoking (never-smoker, ex-smoker, current smoker).

\section{Statistical Analysis}

As within-pair analysis is based on comparing differences within twin pairs, continuous measures of BMI and depressive symptom score from the BDI were used to retain individual variation. However, in order to compare the associations with previous studies, we also ran analyses using dichotomized measures of obesity (BMI $\geq 30 \mathrm{~kg} / \mathrm{m}^{2}$ ) and BDI caseness (BDI score $\geq 10$, indicating at least mild depression). In these analyses, we used logistic regression to derive odds ratios with accompanying 95\% confidence intervals. The measure of negative mood in the MIDUS study was not dichotomized due to lack of established cut-offs.

Preliminary analyses of data from the Finnish Twin Cohort showed that mean BMI was $23 \mathrm{~kg} / \mathrm{m}^{2}$ among nonobese individuals and $33 \mathrm{~kg} / \mathrm{m}^{2}$ among obese individuals. The linear regression coefficients were calculated for a 10unit increase in BMI to reflect this mean difference in BMI between the non-obese and obese groups. All models were adjusted for sex, age, education, and smoking.

We used within-pair comparison among MZ and DZ twins to examine whether the overall association based on standard regression modeling was replicated in within-twin pair analysis; that is, whether the twin with a higher BMI had higher depressive symptoms than his/her co-twin with a lower BMI (Carlin et al., 2005). In the Finnish Twin Cohort, Tobit regression with floor-censoring was used to take into 


\section{TABLE 1}

Characteristics of Study Participants of the Two Cohort Studies

\begin{tabular}{|c|c|c|}
\hline & $\begin{array}{l}\text { Finnish twin } \\
\text { cohort } \\
(n=8,224)\end{array}$ & $\begin{array}{l}\text { MIDUS twin } \\
\text { sample } \\
(n=1,105)\end{array}$ \\
\hline \multicolumn{3}{|l|}{ Sex } \\
\hline Men & $42.4(3,488)$ & $43.6(482)$ \\
\hline Women & $57.6(4,736)$ & $56.4(623)$ \\
\hline Age (years) $\dagger$ & $44.1(7.8)$ & $45.1(12.3)$ \\
\hline Body mass index $\left(\mathrm{kg} / \mathrm{m}^{2}\right) \dagger$ & $24.5(3.7)$ & $26.2(5)$ \\
\hline Obese $(\mathrm{BMI} \geq 30)$ & $7.3(603)$ & $20.9(231)$ \\
\hline Depressive symptoms (BDI score) $\dagger$ & $5.30(5.89)$ & - \\
\hline Negative mood $\dagger$ & - & $49(9.8)$ \\
\hline \multicolumn{3}{|l|}{ Smoking } \\
\hline Never-smoker & $50.0(4,108)$ & $52.5(580)$ \\
\hline Ex-smoker & $22.3(1,835)$ & $26.5(293)$ \\
\hline Current smoker & $27.7(2,281)$ & $21.0(232)$ \\
\hline \multicolumn{3}{|l|}{ Education } \\
\hline Primary & $35.9(2,955)$ & 9.7 (107) \\
\hline Secondary & $53.2(4,376)$ & $61.6(679)$ \\
\hline Tertiary & $10.9(893)$ & $28.7(316)$ \\
\hline
\end{tabular}

account the positive skewness in the distribution of BDI depressive symptoms.

In addition, we fitted bivariate twin models using Cholesky decomposition to examine whether the association between BMI and depressive symptoms was mediated by common genes, shared environment, or non-shared environment. To take into account the possibility that health conditions leading to weight loss and underweight might bias the association between BMI and depressive symptoms, we repeated the main analyses in subsamples first excluding twin pairs with either twin having BMI less than $20 \mathrm{~kg} / \mathrm{m}^{2}$ and then twin pairs with BMI less than $22 \mathrm{~kg} / \mathrm{m}^{2}$. These cutoffs were arbitrary and were selected only for the purpose of sensitivity analysis.

\section{Results}

\section{The Finnish Twin Cohort}

The baseline characteristics of the Finnish Twin Cohort study members are shown in Table 1. Obesity was associated with 1.53 -fold $(95 \% \mathrm{CI}=1.21,1.92)$ increased odds of mild depression in all individuals, the point estimates being 1.78fold in MZ individuals versus 1.42 in DZ twins (Table 2). These estimates changed little after excluding participants with particularly low BMI from the analysis.

Repeating this analysis with continuous measures of BMI and depressive symptoms showed similar results although the positive association between BMI and depression score was substantially stronger when people with BMI $<20 \mathrm{~kg} / \mathrm{m}^{2}$ or BMI $<22 \mathrm{~kg} / \mathrm{m}^{2}$ were removed from the analysis. This strengthening linear association after the exclusion of individuals with low BMI was because individuals with BMI less than 20 had higher levels of depressive symptoms $(\mathrm{B}=0.82,95 \% \mathrm{CI}=0.33,1.30)$ compared to individuals with BMI between 20 and 25 .

In DZ twins, the within-pair analysis produced very similar estimates as the standard regression analysis (Table 2), suggesting that the association between higher BMI and higher depressive symptoms was unlikely to be confounded by factors shared by DZ twin pairs. In contrast, in MZ twin pairs there was no within-pair association between BMI and depressive symptoms. After excluding individuals with BMI below $20 \mathrm{~kg} / \mathrm{m}^{2}$, there was no overlap between the point estimate of the overall association and the confidence interval of the within-pair association, indicating that these estimate were different at $p=.05$.

Figure 1 shows results from the bivariate quantitative genetic model. There was a positive association between BMI and depressive symptoms, and the majority of this association was attributable to genetic influences, as only this pathway was statistically significant. The genetic correlation indicated $12 \%$ overlap in the shared genetic background of BMI and depressive symptoms $(r=0.12,95 \% \mathrm{CI}=0.06$, 0.17; see Online Supplementary Material Appendix 1 and eTable 1 for details of the genetic correlation and eFigure 1 for the best-fitting genetic model).

\section{MIDUS}

The prevalence of obesity was three times as high in the MIDUS sample compared to Finnish Twin Cohort (21\%

TABLE 2

Twin-Pair Analysis of BMI and Depressive Symptoms, Adjusted for Age, Sex, Education, and Smoking: The Finnish Twin Cohort

\begin{tabular}{|c|c|c|c|c|c|c|c|}
\hline \multirow[b]{2}{*}{ Population } & \multicolumn{3}{|c|}{ Depression (dichotomous measure) } & \multicolumn{3}{|c|}{ Depression score (continuous measure) } & \multirow[b]{2}{*}{ Total $N$ (full pairs) } \\
\hline & $O R^{*}[95 \% \mathrm{Cl}]$ & $p$ & Overall $\mathrm{B} \uparrow[95 \% \mathrm{Cl}]$ & $p$ & Within-pair $\mathrm{B} \dagger[95 \% \mathrm{Cl}]$ & $p$ & \\
\hline \multicolumn{8}{|l|}{ MZ twins } \\
\hline All & $1.78[1.1,2.9]$ & 0.03 & $0.90[0.1,1.7]$ & 0.03 & $-0.17[-1.6,1.3]$ & 0.50 & $2846(1324)$ \\
\hline $\mathrm{BMI}>20 \mathrm{~kg} / \mathrm{m}^{2}$ & $1.83[1.1,2.9]$ & 0.02 & $1.69[0.8,2.5]$ & $<0.001$ & $-0.33[-1.9,1.2]$ & 0.39 & $2580(1133)$ \\
\hline $\mathrm{BMI}>22 \mathrm{~kg} / \mathrm{m}^{2}$ & $1.78[1.1,2.9]$ & 0.02 & $2.01[1.0,3.0]$ & $<0.001$ & $0.03[-1.9,2.0]$ & 0.86 & $2022(817)$ \\
\hline \multicolumn{8}{|l|}{ DZ twins } \\
\hline All & $1.42[1.1,1.8]$ & 0.08 & $0.73[0.2,1.3]$ & 0.01 & $0.86[0.0,1.7]$ & 0.08 & $5369(2435)$ \\
\hline $\mathrm{BMI}>20 \mathrm{~kg} / \mathrm{m}^{2}$ & $1.44[1.1,1.9]$ & 0.06 & $0.99[0.4,1.6]$ & 0.001 & $1.27[0.3,2.2]$ & 0.02 & 4986 (2128) \\
\hline $\mathrm{BMI}>22 \mathrm{~kg} / \mathrm{m}^{2}$ & $1.42[1.1,1.9]$ & 0.07 & $1.17[0.5,1.9]$ & 0.001 & $1.47[0.4,2.6]$ & 0.02 & 4041 (1501) \\
\hline
\end{tabular}

Note: *Odds ratio for obese $\left(\mathrm{BMI}>30 \mathrm{~kg} / \mathrm{m}^{2}\right)$ versus non-obese $\left(\mathrm{BMI}<30 \mathrm{~kg} / \mathrm{m}^{2}\right)$ with dichotomous depression as the outcome.

$\dagger \mathrm{B}$ for 10-unit increase in $\mathrm{BM}$ l with continuous depression score as the outcome. Overall regressions are fitted with random-intercept Tobit models, within-pair regression are fitted using fixed-effect estimator to compare twin pairs with each other. Only pairs with full data for each member of twin pair are included in the within-pair analysis. 
TABLE 3

Twin-Pair Analysis of BMI in Relation to Negative Mood (Adjusted for Age, Sex, Education, and Smoking): The MIDUS Twin Sample

\begin{tabular}{|c|c|c|c|c|c|}
\hline \multirow[b]{2}{*}{ Population } & \multicolumn{4}{|c|}{ Negative mood score } & \multirow[b]{2}{*}{ Total $N$ (full pairs) } \\
\hline & Overall $\mathrm{B} \dagger[95 \% \mathrm{Cl}]$ & $p$ value & Within-pair $\mathrm{B} \dagger[95 \% \mathrm{Cl}]$ & $p$ value & \\
\hline \multicolumn{6}{|l|}{$M Z$ twins } \\
\hline All & $2.66[0.8,4.5]$ & 0.01 & $-0.67[-4.2,2.9]$ & 0.71 & $589(293)$ \\
\hline $\mathrm{BMI}>20 \mathrm{~kg} / \mathrm{m}^{2}$ & $2.95[1.0,4.9]$ & 0.003 & $-0.55[-4.3,3.2]$ & 0.77 & $560(274)$ \\
\hline $\mathrm{BMI}>22 \mathrm{~kg} / \mathrm{m}^{2}$ & $3.50[1.4,5.6]$ & 0.001 & $-1.03[-4.9,2.9]$ & 0.60 & $500(234)$ \\
\hline \multicolumn{6}{|l|}{ DZ twins } \\
\hline All & $1.26[-0.4,2.9]$ & 0.71 & $1.70[-0.9,4.3]$ & 0.20 & $514(257)$ \\
\hline $\mathrm{BMI}>20 \mathrm{~kg} / \mathrm{m}^{2}$ & $1.37[-0.4,3.1]$ & 0.77 & $1.66[-1.1,4.4]$ & 0.24 & $489(233)$ \\
\hline $\mathrm{BMI}>22 \mathrm{~kg} / \mathrm{m}^{2}$ & $0.74[-1.2,2.6]$ & 0.60 & $1.67[-1.2,4.6]$ & 0.26 & $431(190)$ \\
\hline
\end{tabular}

A

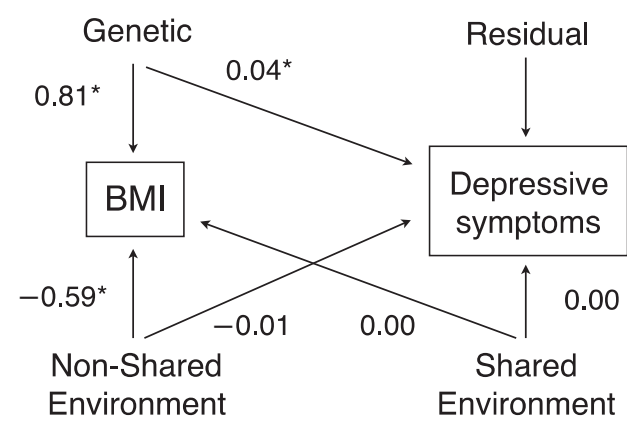

B

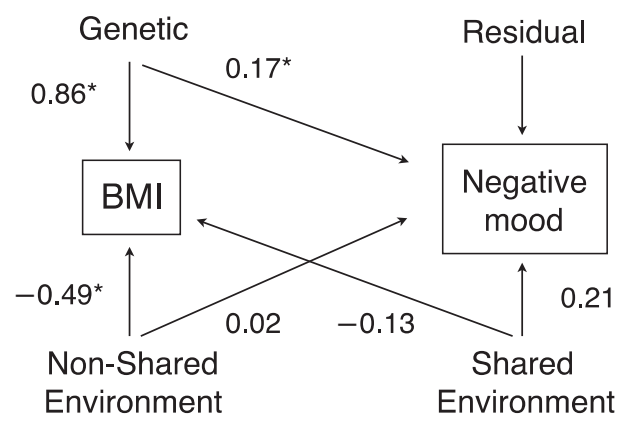

\section{FIGURE 1}

Bivariate genetic model (Cholesky decomposition) of the association between body mass index (BMI) and depressive symptoms into pathways mediated by genetic factors, shared environment, and non-shared environment in the Finnish Twin Cohort (panel $A ; n=1,329$ MZ twin pairs; $n=2,439 \mathrm{DZ}$ pairs) and MIDUS twin sample (panel $B ; n=293 \mathrm{MZ}$ twin pairs; $n=257 \mathrm{DZ}$ pairs). Values are standardized regression coefficients $(S D=1)$, with statistically significant pathways marked with an asterisk $\left({ }^{*} p<.05\right)$. Panel A shows the model for the total sample ( $n=1,329 \mathrm{MZ}$ twin pairs; $n=2,439 \mathrm{DZ}$ pairs). See Supplementary Figure 1 in the Online Supplementary Material for the best-fitting models.

vs. $7 \%$ ). Compared to normal-weight participants of the total twin sample of MIDUS (BMI between $18.5 \mathrm{~kg} / \mathrm{m}^{2}$ and $\left.25 \mathrm{~kg} / \mathrm{m}^{2}\right)$, higher levels of negative mood were observed in overweight $(\mathrm{B}=0.94, \mathrm{CI}=-0.34,2.22 ; p=.15)$ and obese participants $(\mathrm{B}=2.38, \mathrm{CI}=0.72,4.03 ; p=.005)$ but not in underweight participants $(\mathrm{B}=0.00, \mathrm{CI}=-3.61,3.60$; $p=.99)$, with a linear trend of $\mathrm{B}=1.99(\mathrm{CI}=0.78,3.20$; $p=.001)$. Thus, the exclusion of participants with low BMI did not have as strong influence on the coefficients as in the Finnish sample.

The total and within-pair regression analyses in the MIDUS sample replicated the results of the Finnish study by showing that the positive association between BMI and negative mood observed in MZ twins was not observed in within-pair analysis (Table 3). The associations were weaker in DZ than in MZ twins, but there were no marked differences between total versus within-pair regressions among DZ twins.

The quantitative genetic models also supported the results of the Finnish study as the association between BMI and negative mood supported the genetic pathway but not the environmental pathways (Figure 1). The genetic correlation indicated $20 \%$ overlap in the shared genetic background between BMI and negative mood $(r=0.20,95 \% \mathrm{CI}$ $=0.08,0.31$; see Online Supplementary Material Appendix 1 and Supplementary Table S1 for details of the genetic correlation and Supplementary Figure S1 for the best-fitting genetic model). The results were similar when negative and positive affect scales were used as two separate outcomes instead of a single scale (Supplementary Table S2 and Figure S2).

\section{Discussion}

In two independent cohort studies of $\mathrm{MZ}$ and $\mathrm{DZ}$ twins, higher BMI was associated with higher depressive symptoms, and this association was mediated mostly by shared genetic factors; the association was not observed when comparing $\mathrm{MZ}$ twin pairs with each other.

The main limitation of the present study is the lack of repeated measurements of BMI and depressive symptoms over time. Although the MIDUS study has repeated 
measurements, the number of twin participants was not sufficient for longitudinal analysis (details not shown), and in the Finnish twin cohort, depressive symptoms using the BDI have been assessed only once. Previous results using longitudinal data have suggested a bidirectional association between obesity and depression (Luppino et al., 2010), with each direction mediated by different biological and psychological mechanisms (Kivimäki et al., 2009). Thus, the present results provide evidence on the nature of stable associations between BMI and depressive symptoms but not necessarily about changes over time. Another limitation was the use of self-reported measures only.

Obesity and depressive symptoms may share common pathophysiological pathways, including the HPA axis, immuno-inflammatory reactions and insulin signaling (Capuron et al., 2008; Dixon et al., 2008; Ladwig et al., 2003; Miller et al., 2003; Olszanecka-Glinianowicz et al., 2009; Soczynska et al., 2011). For example, obesity and depression may both represent pro-inflammatory states (Ladwig et al., 2003; Miller et al., 2003), which would be consistent with a shared genetic background of body weight and depressive symptoms. Indeed, several genetic variants related to depression are also related to inflammation (Raison \& Miller, 2013). The obesity-related FTO gene has been associated with both lower (Kivimäki et al., 2011) and higher (Lawlor et al., 2011; Samaan et al., 2013) symptoms of depression and anxiety, while a 31-SNP genetic risk marker for higher BMI was associated with higher depressive symptoms (Jokela et al., 2012). The associations between obesityrelated genes and depressive symptoms in these studies have been largely independent of BMI, suggesting that at least some of the obesity-related genes may be directly related to risk of mental disorders - and not mediated by the phenotypic effects of higher body weight (Samaan et al., 2013). The current findings are in agreement with such a direct genetic association.

Two previous family-based analyses have produced mixed findings on the genetic link between body weight and depressive symptoms (Afari et al., 2010; Choy et al., 2009). In an analysis of $712 \mathrm{MZ}$ and $281 \mathrm{DZ}$ female twin pairs from the Washington twin registry (Afari et al., 2010), $12 \%$ of the genetic component of depression was shared with genetic component of obesity, with no contribution from shared or non-shared environmental factors. By contrast, a Dutch family-based study found no shared genetic factors to account for the association between obesity and depressive symptoms in a pedigree analysis of 1,355 women and 1,028 men (Choy et al., 2009). Our results are in agreement with the findings from the Washington twin study.

The non-genetic associations estimated in the two cohorts were not statistically significant in the within-pair comparison of MZ twins or in the bivariate genetic models. The non-significant associations in the within-pair analyses hinted at a possible inverse association between BMI and depressive symptoms. Additional data are needed to test whether such an inverse association would be observed in larger samples. It must be emphasized that the absence of an environmentally mediated association should not be interpreted to indicate that the environmental risk factors increasing the prevalence of obesity would not influence depressive symptoms over time or between populations (Davey, 2011). The results only suggest that environmentally induced variation in BMI is not associated with differences in depressive symptoms among individuals at a particular time.

In sum, twin-pair analysis and quantitative genetic modeling in two twin studies from Finland and the US provided converging evidence for an association between higher BMI and higher depressive symptoms in adults. This association appears to be explained by a shared genetic background rather than environmental factors, but further research is needed to confirm this finding.

\section{Acknowledgments}

This research was supported by the Academy of Finland (grant numbers: 124,322, 124,271, 132,944, 141,054, 265,240, and 264,146); the Medical Research Council (MRC, K013351); the US National Institutes of Health (R01HL036310; R01AG034454); and the Finnish Work Environment Fund. The Centre for Cognitive Ageing and Cognitive Epidemiology is supported by the Biotechnology and Biological Sciences Research Council, the Engineering and Physical Sciences Research Council, the Economic and Social Research Council, the MRC, and the University of Edinburgh as part of the cross-council Lifelong Health and Wellbeing initiative. G.D.B. is a Wellcome Trust Fellow and M.K. an Economic and Social Research Council Professor.

\section{Supplementary Material}

To view supplementary material for this article, please visit http://dx.doi.org/10.1017/thg.2016.14.

\section{References}

Afari, N., Noonan, C., Goldberg, J., Roy-Byrne, P., Schur, E., Golnari, G., ... Buchwald, D. (2010). Depression and obesity: Do shared genes explain the relationship?. Depression \& Anxiety, 27, 799-806.

Beck, A. T., \& Steer, R. A. (1987). Manual for the Revised Beck Depression Inventory. San Antonio, TX: Psychological Corporation.

Blaine, B. (2008). Does depression cause obesity? A metaanalysis of longitudinal studies of depression and weight control. Journal of Health Psychology, 13, 1190-1197.

Brim, O. G., Baltes, P. B., Bumpass, L. L., Cleary, P. D., Featherman, D. L., Hazzard, W. R. ... Ryff, C. D. (2007). National survey of midlife development in the United States (MIDUS), 1995-1996 (ICPSR02760-v4). Ann Arbor, MI: DataStat, Inc./Boston, MA: Harvard Medical School, Department of Health Care Policy. 
Capuron, L., Su, S., Miller, A. H., Bremner, J. D., Goldberg, J., Vogt, G. J., ... Vaccarino, V. (2008). Depressive symptoms and metabolic syndrome: Is inflammation the underlying link?. Biological Psychiatry, 64, 896-900.

Carlin, J. B., Gurrin, L. C., Sterne, J. A. C., Morley, R., \& Dwyer, T. (2005). Regression models for twin studies: A critical review. International Journal of Epidemiology, 34, 1089-1099.

Choy, W. C., Lopez-Leon, S., Aulchenko, Y. S., Mackenbach, J. R., Oostra, B. A., van Duijn, C. M., ... Cecile, J. W. (2009). Role of shared genetic and environmental factors in symptoms of depression and body composition. Psychiatric Genetics, 19, 32-38.

Crisp, A. H., \& McGuiness, B. (1976). Jolly fat — relation between obesity and psychoneurosis in general population. British Medical Journal, 1, 7-9.

Davey Smith, G. (2011). Epidemiology, epigenetics and the 'Gloomy prospect': Embracing randomness in population health research and practice. International Journal of Epidemiology, 40, 537-562.

Dixon, J. B., Hayden, M. J., Lambert, G. W., Dawood, T., Anderson, M. L., Dixon, M. E., \& O’Brien, P. E. (2008). Raised CRP levels in obese patients: Symptoms of depression have an independent positive association. Obesity, 16, 2010-2015.

Gariepy, G., Nitka, D., \& Schmitz, N. (2010). The association between obesity and anxiety disorders in the population: A systematic review and meta-analysis. International Journal of Obesity, 34, 407-419.

Gariepy, G., Wang, J. L., Lesage, A. D., \& Schmitz, N. (2010). The longitudinal association from obesity to depression: Results from the 12-year national population health survey. Obesity, 18, 1033-1038.

Jokela, M., Elovainio, M., Keltikangas-Jarvinen, L., Batty, G. D., Hintsanen, M., Seppala, I., ... Kivimäki, M. (2012). Body mass index and depressive symptoms: Instrumentalvariables regression with genetic risk score. Genes, Brains, \& Behavior, 11, 942-948.

Kaprio, J., \& Koskenvuo, M. (2002). Genetic and environmental factors in complex diseases: The older finnish twin cohort. Twin Research, 5, 358-365.

Kivimäki, M., Jokela, M., Hamer, M., Geddes, J., Ebmeier, K., Kumari, M., ... Batty, G. D. (2011). Examining overweight and obesity as risk factors for common mental disorder using FTO genotype-instrumented analysis: The Whitehall II study, 1985-2004. American Journal of Epidemiology, 173, 421-429.

Kivimäki, M., Lawlor, D. A., Singh-Manoux, A., Batty, G. D., Ferrie, J. E., Shipley, M. J., ... Jokela, M. (2009). Common mental disorder and obesity: Insight from four repeat measures over 19 years: Prospective Whitehall II cohort study. British Medical Journal, 339, b3765.

Ladwig, K. H., Marten-Mittag, B., Lowel, H., Doring, A., \& Koenig, W. (2003). Influence of depressive mood on the association of CRP and obesity in 3205 middle aged healthy men. Brain, Behavior, and Immunity, 17, 268-275.
Lawlor, D. A., Harbord, R. M., Tybjaerg-Hansen, A., Palmer, T. M., Zacho, J., Benn, M., ... Nordestgaard, B. G. (2011). Using genetic loci to understand the relationship between adiposity and psychological distress: A Mendelian randomization study in the copenhagen general population study of 53221 adults. Journal of Internal Medicine, 269, 525537.

Lawlor, D. A., Hart, C. L., Hole, D. J., Gunnell, D., \& Smith, G. D. (2007). Body mass index in middle life and future risk of hospital admission for psychoses or depression: Findings from the renfrew/paisley study. Psychological Medicine, 37, 1151-1161.

Luppino, F. S., de Wit, L. M., Bouvy, P. F., Stijnen, T., Cuijpers, P., Penninx, B., \& Zitman, F. G. (2010). Overweight, obesity, and depression: A systematic review and meta-analysis of longitudinal studies. Archives of General Psychiatry, 67, 220229.

Markowitz, S., Friedman, M. A., \& Arent, S. M. (2008). Understanding the relation between obesity and depression: Causal mechanisms and implications for treatment. Clinical Psychology-Science and Practice, 15, 1-20.

Miller, G. E., Freedland, K. E., Carney, R. M., Stetler, C. A., \& Banks, W. A. (2003). Pathways linking depression, adiposity, and inflammatory markers in healthy young adults. Brain, Behavior, and Immunity, 17, 276285.

Mroczek, D. K., \& Kolarz, C. M. (1998). The effect of age on positive and negative affect: A developmental perspective on happiness. Journal of Personality and Social Psychology, $75,1333-1349$.

Olszanecka-Glinianowicz, M., Zahorska-Markiewicz, B., Kocelak, P., Janowska, J., Semik-Grabarczyk, E., Wikarek, T., ... Dąbrowski, P. (2009). Is chronic inflammation a possible cause of obesity-related depression?. Mediators of Inflammation, Article ID 439107.

Palinkas, L. A., Wingard, D. L., \& Barrett-Connor, E. (1996). Depressive symptoms in overweight and obese older adults: A test of the 'jolly fat' hypothesis. Journal of Psychosomatic Research, 40, 59-66.

Raison, C. L., \& Miller, A. H. (2013). The evolutionary significance of depression in Pathogen Host Defense (PATHOSD). Molecular Psychiatry, 18, 15-37.

Samaan, Z., Anand, S., Zhang, X., Desai, D., Rivera, M., Pare, G., ... Meyre, D. (2013). The protective effect of the obesity-associated rs9939609 A variant in fat mass- and obesity-associated gene on depression. Molecular Psychiatry, 18, 1281-1286.

Soczynska, J. K., Kennedy, S. H., Woldeyohannes, H. O., Liauw, S. S., Alsuwaidan, M., Yim, C. Y., ... McIntyre, (2011). Mood disorders and obesity: Understanding inflammation as a pathophysiological nexus. Neuromolecular Medicine, 13, 93-116.

Wardle, J., Chida, Y., Gibson, E. L., Whitaker, K. L., \& Steptoe, A. (2011). Stress and adiposity: A meta-analysis of longitudinal studies. Obesity, 19, 771-778. 